\title{
Long-period gratings fabricated in photonic crystal fibre H.Dobb $^{\mathrm{a}}$, J.S.Petrovic ${ }^{\mathrm{a}}$, V.Mezentsev ${ }^{\mathrm{a}}$, D.J.Webb ${ }^{\mathrm{a}}$ and K.Kalli ${ }^{\mathrm{b}}$
}

\author{
${ }^{a}$ Photonics Research Group, Aston University, Aston Triangle, Birmingham, B4 7ET, UK. \\ ${ }^{\mathrm{b}}$ Higher Technical Institute, C. Kavafi Str., Aglantzia, P.O. Box 20423, 2152 Nicosia, Cyprus.
}

\begin{abstract}
Long period gratings in two types of photonic crystal fibre have been studied. The gratings display negligible temperature sensitivity but useful sensitivity to other measurands. Theoretical modelling suggests that unusual phase matching conditions apply.
\end{abstract}

Keywords: Photonic Crystal Fibre, long-period gratings

\section{INTRODUCTION}

Long- period gratings (LPGs) consist of a large-scale $(100 \mu \mathrm{m}$ to $1 \mathrm{~mm})$ periodic axial perturbation in the core of a single mode optical fibre. The effect of this modulation is to couple light from the fundamental core mode to co-propagating cladding modes leading to a series of attenuation bands in the transmission spectrum. A necessary condition of such a resonance is phase matching between these modes, when the intermodal beat period equals the grating period. LPGs are of interest for sensing applications due to spectral shifts of the attenuation bands occurring as a result of their sensitivity to several external measurands: temperature, strain, curvature and surrounding refractive index ${ }^{1-2}$.

One of the persistent problems associated with using LPGs as sensors is their cross-sensitivity to several measurands especially temperature, which results in discriminatory schemes needing to be employed to separate the effect of temperature from the desired measurands. Since the LPG's sensitivity to measurands depends on the waveguide and material dispersion of the fibre ${ }^{3}$, different optical fibre geometries and compositions can increase or decrease the LPG sensitivity. Photonic Crystal Fibre (PCF) has the potential for such optimisation due to its dispersion properties being strongly dependent on the air-hole geometry. Recently, it has been shown that LPGs can be produced in nonphotosensitive PCF by periodically collapsing the holes by heat treatment with a $\mathrm{CO}_{2}$ laser ${ }^{4}$ or by using an electric arc to modify the fibre structure ${ }^{5}$.

In this paper we report on studies of LPGs fabricated in two different types of PCF, using the electric arc technique. The results are compared with a comprehensive eigenmode analysis of the guided modes supported by the PCF with modified phase matching conditions. We have compared a modelled dispersion map with the measured transmission spectra and found good agreement on the positions of the resonant modes. All the significant features of the observed transmission spectra are explained by multiple-period resonances between cladding modes, supported by the bulk silica cladding, and a core mode.

\section{ENDLESSLY SINGLE MODE PCF}

The first endlessly single mode (ESM) PCF (Blaze Photonics, ESM-1550-01) was made from pure silica and had a core diameter of $12 \mu \mathrm{m}$ surrounded by 54 air holes, with the space between adjacent holes being $8 \mu \mathrm{m}$. This particular PCF has a relatively thick bulk silica cladding (outer diameter $125 \mu \mathrm{m}$ ) separated from the central core by the four rings of air holes. Using the electric arc technique two LPGs with different periods were fabricated; one with a period of $500 \mu \mathrm{m}$ and the other $600 \mu \mathrm{m}$. The resulting spectra can be seen in Fig 1. The $1409 \mathrm{~nm}$ attenuation band of the $500 \mu \mathrm{m}$ period LPG was experimentally investigated for its spectral sensitivity to external measurands since this band was of a significant strength and at a suitable wavelength.

\subsection{Experimental Results}

The temperature sensitivity was investigated by placing the LPG on an insulated Peltier heater and varying the temperature from $20.0^{\circ} \mathrm{C}$ to $90.5^{\circ} \mathrm{C}$. This produced no measurable change in the central wavelength of the attenuation band. From the measurement accuracy we have deduced a temperature sensitivity of $\mathrm{d} \lambda / \mathrm{dT}=0 \pm 10 \mathrm{pm} /{ }^{\circ} \mathrm{C}$. This may be compared with the results of Humbert et al. ${ }^{6}$ who quote a temperature sensitivity of $9 \mathrm{pm} /{ }^{\circ} \mathrm{C}$ in the range of $25-160^{\circ} \mathrm{C}$ for a similar structured PCF.

The strain sensitivity of the $1409 \mathrm{~nm}$ attenuation band was investigated by fixing one end of the grating on a block and the other to a translation stage. The effect of the strain on the attenuation band is shown in Fig. 2. The resulting wavelength shift gives a strain sensitivity of $\mathrm{d} \lambda / \mathrm{d} \sigma=-2.0 \pm 0.1 \mathrm{pm} / \mu \varepsilon$. The negative value of the sensitivity indicates a 


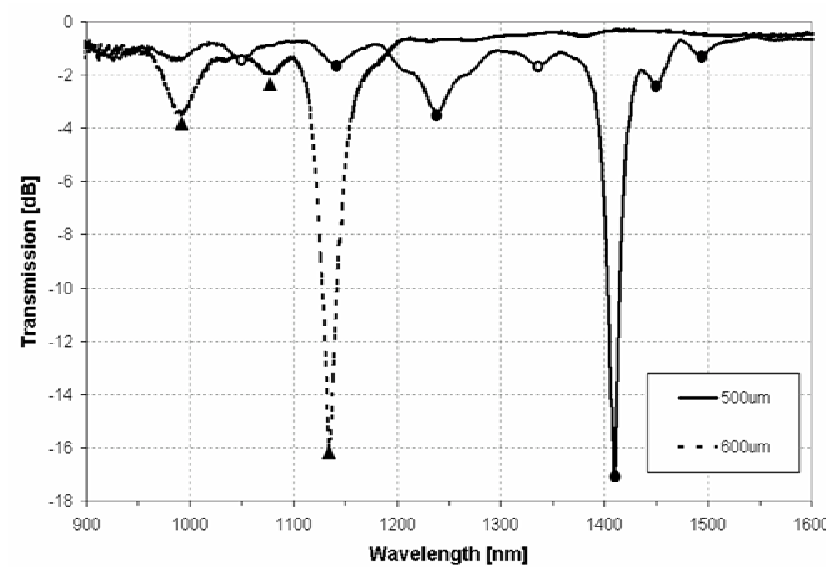

Fig.1. Transmission spectra for different grating periods. Solid line $-500 \mu \mathrm{m}$, dashed line $-600 \mu \mathrm{m}$. The attenuation bands associated with multiple period resonances are marked with circles $(500 \mu \mathrm{m})$ and triangles $(600 \mu \mathrm{m})$.

Full circles - double period resonances; hollow circles - triple and quadruple period resonances.

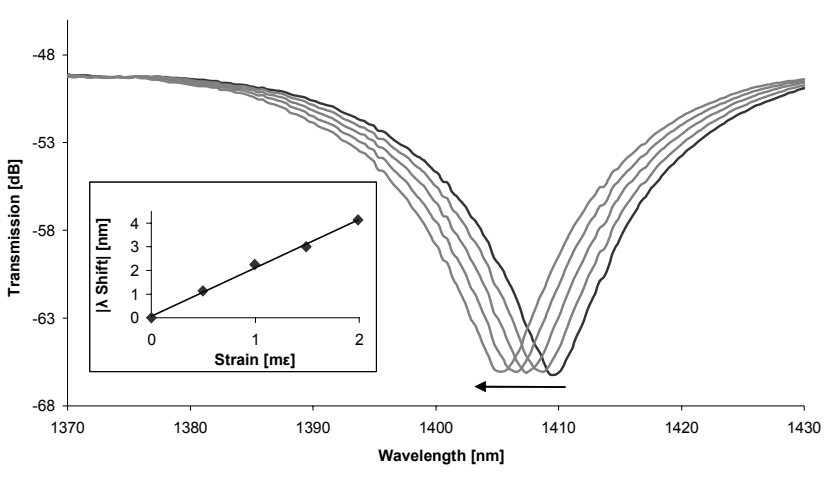

Fig. 2 Spectral response of LPG to strain in range $0-1.98 \mathrm{~m} \varepsilon$. Unstrained curve in bold.

Inset: Wavelength shift as a result of applied strain, showing straight line fit with $\mathrm{d} \lambda / \mathrm{d} \sigma=-2.0 \pm 0.1 \mathrm{pm} / \mu \varepsilon$.

blue wavelength shift with increasing strain. The blue wavelength shift occurs because the applied strain causes an increase in the grating period. As demonstrated in fig 1, increasing the grating period in PCF causes the location of the LPG attenuation bands to shift to lower wavelengths ${ }^{6}$.

\subsection{Theoretical Modelling}

Guided modes and corresponding propagation constants and effective refractive indices were found by means of a fullvectorial Maxwell finite element solver bundled with the commercial package FEMLAB. Eigenmodes were two-, four-, or six-fold degenerated corresponding to different polarisations and the six-fold symmetry of the problem. This geometry supports a large number of cladding modes similar to those of a ring resonator formed by the bulky cladding ring. It turns out that the mode localised in the core is not the fundamental mode for such a waveguide and its order is 148 . The lowest order modes (i.e. those with the highest effective refractive indices) are actually cladding modes, as the large cladding region allows for more spacious modes. There is a correspondence between the mode order and mode spatial finesse the higher the order the finer is the spatial structure of the mode. The relatively small size of the core determines the fairly high order of the core-guided mode. The effective refractive index decreases with the mode order so that the effective indices of many cladding modes exceed that of the core mode as shown in Fig 3a. Such behaviour stems from the fact that cladding modes propagate through the pure silica cladding and their interaction with the holey cladding is weaker compared to that of the core mode.

\subsubsection{Multiple Period Resonances}

In order to identify modes responsible for resonant coupling to a core mode, the usual phase matching condition, $L=2 \pi\left|\beta_{c o}-\beta_{c l}\right|$ was applied, where $L$ is a beating period, $\beta_{c o}$ and $\beta_{c l}$ are the propagation constants of core and cladding modes. Typically, one LPG period $\Lambda=L$ is enough to accumulate the phase difference of $2 \pi$ required for phase matching. However, in the considered cases a single grating period is not enough to provide phase matching with any of the cladding modes for wavelengths up to $2 \mu \mathrm{m}$. Phase matching can only be achieved after longer propagation i.e. the beat length should be a multiple of the grating period. The results of this phase matching analysis are presented in Fig $3 \mathrm{~b}$. It shows the beat length $L=2 \pi\left|\beta_{c o}-\beta_{c l}\right|$ as a function of wavelength and horizontal lines correspond to double the periods of the two gratings studied experimentally, which had periods of 500 and 600 microns. These results confirm the experimental observation that gratings with longer periods have blue shifted resonances. Fig. 4 shows the intensity profiles of the modes corresponding to multiple period resonances for the LPG with a period of $500 \mu \mathrm{m}$. 


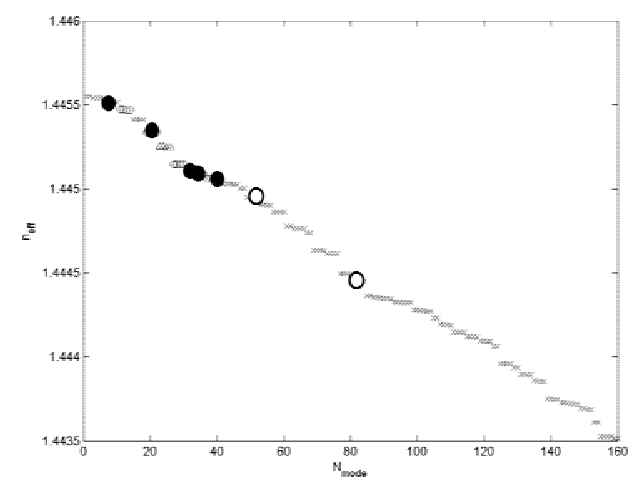

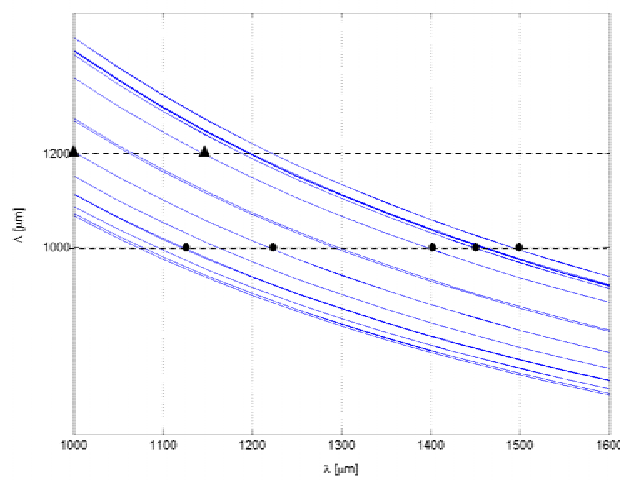

Fig 3. Guided modes supported by PCF: a) Effective refractive index versus mode order b) Dispersion of LPG beat length required for phase matching $L(\lambda)=2 \pi\left|\beta_{\mathrm{co}}-\beta_{\mathrm{cl}}\right|$ for the lowest 40 modes. Circle and triangle marks correspond to those in Fig 1.

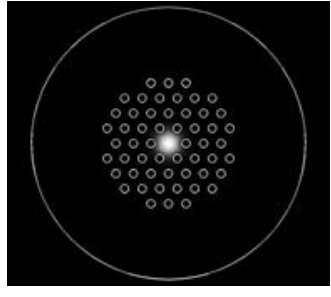

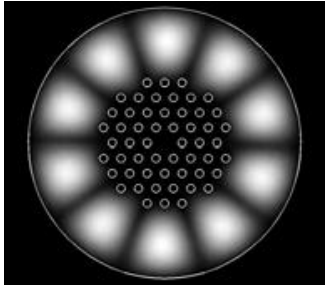

b

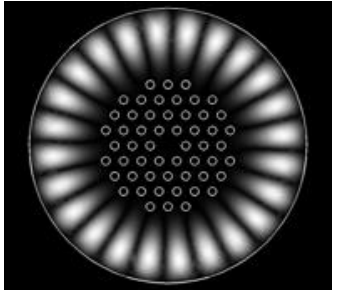

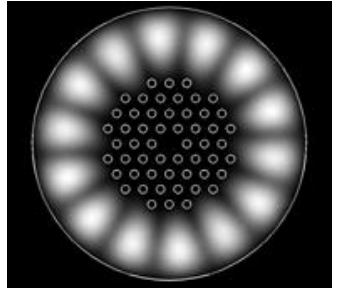

d

Fig 4. Intensity profiles of the resonant modes corresponding to multiple period resonances for an LPG with $\Lambda=500 \mu \mathrm{m}$. Full and hollow circles correspond to those in Fig 1:

a) core mode at $\lambda=1400 \mathrm{~nm}, \mathbf{b}) \bullet 20^{\text {th }}$ mode at $\left.\lambda=1239 \mathrm{~nm}, \mathbf{c}\right) \circ-$ quad period resonance $81^{\text {st }}-$ mode at $\left.\lambda=1338 \mathrm{~nm}, \mathbf{d}\right) \bullet 27^{\text {th }}$ mode at $\lambda=1409 \mathrm{~nm}$

\section{LARGE MODE AREA PCF}

The second ESM PCF investigated had a large-mode area (Crystal Fiber, LMA 10). This PCF had a core diameter of $11 \mu \mathrm{m}$ and was surrounded by 90 air holes with a separation distance of $7.1 \mu \mathrm{m}$. Two LPGs, with periods of $400 \mu \mathrm{m}$ and $500 \mu \mathrm{m}$, were fabricated in this fibre using the same technique as before, see Fig. 5.

\subsection{Experimental Results.}

The $400 \mu \mathrm{m}$ period LPG was investigated for its spectral sensitivity to external measurands. This grating had four attenuation bands located at $1668 \mathrm{~nm}, 1511 \mathrm{~nm}, 1434 \mathrm{~nm}$ and $1389 \mathrm{~nm}$. Investigating the temperature sensitivity, again produced no measurable change in the central wavelength of any of the attenuation bands, from which we deduce a temperature sensitivity of $\mathrm{d} \lambda / \mathrm{dT}=0 \pm 10 \mathrm{pm} /{ }^{\circ} \mathrm{C}$. Investigating the strain sensitivity produced a linear blue wavelength shift of all the attenuation bands. The attenuation band centred at $1668 \mathrm{~nm}$ had a strain sensitivity of $\mathrm{d} \lambda / \mathrm{d} \sigma=-2.5 \pm 0.04$ $\mathrm{pm} / \mu \varepsilon$. The other attenuation bands were also investigated and experienced roughly the same strain sensitivity, except for the band centred at $1389 \mathrm{~nm}$ which had a sensitivity of $\mathrm{d} \lambda / \mathrm{d} \sigma=-1.8 \pm 0.2 \mathrm{pm} / \mu \varepsilon$.

This fibre was also investigated for its bend sensitivity. The fibre was placed in two rotational stages which were mounted on to blocks; one of which was fixed to a translation stage that was moved inwards, thereby bending the fibre downward. To investigate whether the fibre had any directional curvature dependence, the fibre was bent with the rotational stages set to various orientations. The attenuation bands were found to be directionally sensitive with maximum red and blue shifts in the central wavelength observed in two diametrically opposite directions, see table 1 . The band centred at $1668 \mathrm{~nm}$ was found to have the maximum sensitivity to bending with a value of $\mathrm{d} \lambda / \mathrm{dR}=9.6 \pm 1.0$ $\mathrm{nm} . \mathrm{m}$ with the fibre in the $180^{\circ}$ rotational state and a sensitivity of $\mathrm{d} \lambda / \mathrm{dR}=-12.4 \pm 1.2 \mathrm{~nm} . \mathrm{m}$ when in the $0^{\circ}$ position. The negative signs indicate blue wavelength shifts. 


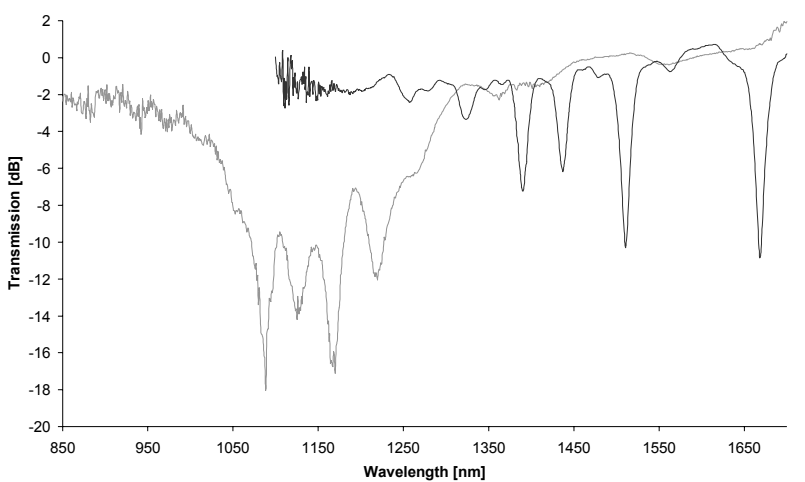

Fig. 5. Transmission spectra of LPGs fabricated in Crystal

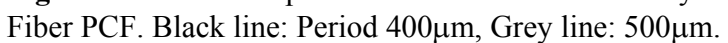

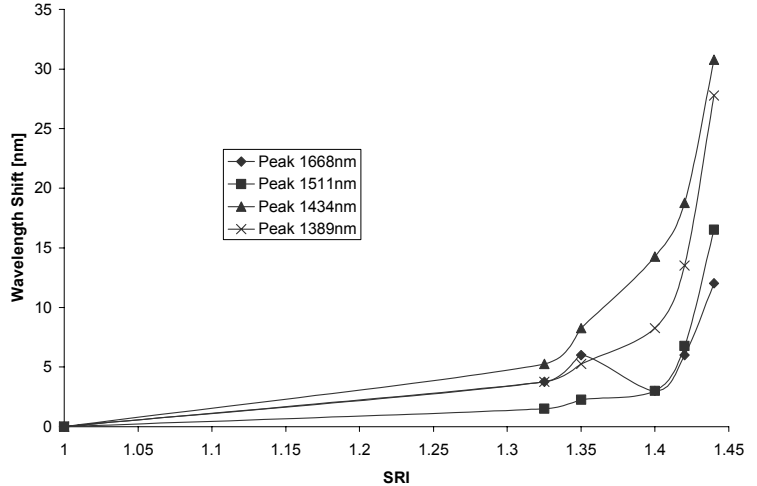

Fig. 6. The wavelength shift of the four attenuation bands as a function of the SRI (less than silica).

\begin{tabular}{|l|c|c|c|c|}
\hline & Atten. Band $1668 \mathrm{~nm}$ & Atten. Band $1511 \mathrm{~nm}$ & Atten. Band $1434 \mathrm{~nm}$ & Atten. Band 1389nm \\
\hline $0^{\circ}$ rotation & $-12.4 \pm 1.2 \mathrm{~nm} . \mathrm{m}$ & $-1.1 \pm 0.1 \mathrm{~nm} . \mathrm{m}$ & $6.5 \pm 0.3 \mathrm{~nm} . \mathrm{m}$ & $4.5 \pm 0.4 \mathrm{~nm} . \mathrm{m}$ \\
\hline $180^{\circ}$ rotation & $9.6 \pm 1.0 \mathrm{~nm} . \mathrm{m}$ & $0.7 \pm 0.1 \mathrm{~nm} . \mathrm{m}$ & $-4.0 \pm 0.5 \mathrm{~nm} . \mathrm{m}$ & $-4.3 \pm 0.5 \mathrm{~nm} . \mathrm{m}$ \\
\hline
\end{tabular}

Table 1. Table showing the spectral responses of all the attenuation bands to the induced curvature

The spectral sensitivity to surrounding refractive index was investigated by placing the LPG in a V-groove and immersing the grating in certified refractive index (CRI) liquids (supplied by Cargille laboratories Inc.), which have a quoted accuracy of \pm 0.0002 . Several different CRI liquids were compared in the range $n=1.325$ to $n=1.514$ ensuring the response of the grating to refractive indices less than and greater than that of silica were studied. For values of CRI less than silica, all the attenuation bands experienced a red wavelength shift. The sensitivity increases markedly, see Fig. 6, until the index matches that of the silica when no co-propagating modes are supported due to an effective infinite cladding. For indices greater than that of silica, the attenuation bands reappear due to weakly guided modes, growing in strength with increasing values of CRI. This LPG behaviour is similar to that observed in $\mathrm{SMF}^{1}$.

\section{CONCLUSIONS}

LPGs in two different types of PCF have been investigated. Both fibres have shown negligible temperature sensitivity whilst having significant sensitivity to other measurands. This is an important factor, since one of the persistent problems of using LPGs as sensors is their cross-sensitivity to temperature, which results in discriminatory schemes needing to be employed to separate the effect of temperature from the desired measurands. Similar strain sensitivity was observed with the two fibres. The Crystal Fiber PCF was also found to have directional bend sensitivity with its maximum sensitivity being $\mathrm{d} \lambda / \mathrm{dR}=-12.4 \pm 1.2 \mathrm{~nm} . \mathrm{m}$; as well as sensitivity to external refractive index. Eigenmode analysis of the guided modes supported by the fibres showed unusual phasematching conditions that may require multiple grating periods for the resonant coupling. Good agreement with the experimentally observed transmission spectra is seen.

\section{REFERENCES}

1. S.W. James and R.T. Tatam. "Optical fibre long-period grating sensors: characteristics and application." Meas. Sci, Technol., 14 (5) pp. R51-R61, (2003)

2. T. Allsop, D.J. Webb and I. Bennion. "A comparison of the sensing characteristics of long period gratings written in three different types of fiber." Opt. Fiber Tech. 4 (9), pp.210-223, (2003)

3. A.M. Vengsarkar, P.J. Lemaire, J.B. Judkins, V. Bhatia, T. Erdogan and J.E. Sipe. "Long-period fiber gratings as band rejection filters." J. Lightwave Technol., 14 (1) pp. 58-64, (1996)

4. D.D. Davies T.K. Gaylord, E.N. Glytsis, S.G. Kosinski, S.C. Mettler and A.M. Vengsarkar. "Long-period fibre grating fabrication with focused $\mathrm{CO}_{2}$ laser pulses." Electron. Lett., 34 (3) pp. 302-303, (1998)

5. G. Humbert, A. Malki, S. Février, P. Roy and D. Pagnoux. "Electric arc-induced long-period gratings in Ge-free air-silica microstructure fibres." Electron. Lett., 39 (4) pp. 349-350, (2003)

6. K. Morishita and Y. Miyake. "Fabrication and resonance wavelength of long-period gratings in a pure-silica photonic crystal fiber by the glass structure change." J. Lightwave Technol., 22 (2) pp. 625-630, (2004) 